\title{
Crystalline-to-plastic phase transitions in molecularly thin $n$-dotriacontane films adsorbed on solid surfaces
}

\author{
Edgardo A. Cisternas, ${ }^{1}$ Tomás P. Corrales, ${ }^{1}$ Valeria del Campo, ${ }^{1}$ Pamela A. Soza, ${ }^{1}$ \\ Ulrich G. Volkmann, ${ }^{1, a)}$ Mengjun Bai, ${ }^{2}$ Haskell Taub, ${ }^{2}$ and Flemming Y. Hansen ${ }^{3}$ \\ ${ }^{1}$ Facultad de Física, Pontificia Universidad Católica de Chile, Santiago 22, Chile \\ ${ }^{2}$ Department of Physics and Astronomy and University of Missouri Research Reactor, \\ University of Missouri, Columbia, Missouri 65211, USA \\ ${ }^{3}$ Department of Chemistry, Technical University of Denmark, IK 207 DTU, DK-2800 Lyngby, Denmark
}

(Received 5 March 2009; accepted 10 August 2009; published online 21 September 2009)

\begin{abstract}
Crystalline-to-rotator phase transitions have been widely studied in bulk hydrocarbons, in particular in normal alkanes. But few studies of these transitions deal with molecularly thin films of pure $n$-alkanes on solid substrates. In this work, we were able to grow dotriacontane $\left(n-\mathrm{C}_{32} \mathrm{H}_{66}\right)$ films without coexisting bulk particles, which allows us to isolate the contribution to the ellipsometric signal from a monolayer of molecules oriented with their long axis perpendicular to the $\mathrm{SiO}_{2}$ surface. For these submonolayer films, we found a step in the ellipsometer signal at $\sim 331 \mathrm{~K}$, which we identify with a solid-solid phase transition. At higher coverages, we observed additional steps in the ellipsometric signal that we identify with a solid-solid phase transition in multilayer islands $(\sim 333 \mathrm{~K})$ and with the transition to the rotator phase in bulk crystallites $(\sim 337 \mathrm{~K})$, respectively. After considering three alternative explanations, we propose that the step upward in the ellipsometric signal observed at $\sim 331 \mathrm{~K}$ on heating the submonolayer film is the signature of a transition from a perpendicular monolayer phase to a denser phase in which the alkane chains contain on average one to two gauche defects per molecule. () 2009 American Institute of Physics. [doi:10.1063/1.3213642]
\end{abstract}

\section{INTRODUCTION}

Normal alkanes are very useful as prototypes of more complex molecules as well as being the main constituents of thin films of interest in a variety of areas (lubrication, coatings, and membranes). They are also of fundamental interest due to their relative simplicity and wide range of phenomena, including rotator phases, ${ }^{1}$ surface frozen layers, ${ }^{2,3}$ and delayering transitions. ${ }^{4}$

The rotator phases of the $n$-alkanes are typically layered crystals with long-range positional order of the molecules in three dimensions but without long-range order in the rotational degree of freedom of the molecules about their long axis. ${ }^{1}$ Several rotator phases have been identified for bulk alkane crystals, which depend on the molecular length and also on whether the number of carbon atoms is even or odd. ${ }^{1,5}$ For the particular case of dotriacontane $\left(n-\mathrm{C}_{32} \mathrm{H}_{66}\right.$ hereafter denoted as $\mathrm{C} 32)$, has been only one rotator transition reported below its bulk melting temperature $\left(T_{b}\right.$ $=341 \mathrm{~K})$. This transition takes place at a temperature of $\sim 337 \mathrm{~K}$ from a monoclinic crystal to an $R_{\mathrm{III}}$ phase that has a hexagonal triclinic $A B C$ stacking. ${ }^{1,5,6}$ Although rotator phases have been studied in bulk crystals of alkanes, there are few reports of these phases in molecularly thin films. $^{7-11,16}$

Solid C32 films grown on $\mathrm{SiO}_{2}$ surfaces have been found to follow the Stranski-Krastanov growth mode. At the substrate-alkane interface, there is a complete bilayer of mol-

${ }^{a)}$ Electronic mail: volkmann@fis.puc.cl. ecules with their long axis parallel to the surface. ${ }^{12}$ On top of this bilayer, molecules with their long axis perpendicular to the surface coexist with monoclinic and orthorhombic bulk particles. ${ }^{13,14}$ The mesa-shaped orthorhombic particles are surrounded by an area depleted of perpendicular layers. ${ }^{4,15}$

Moreover, Bai et $a l .{ }^{4}$ have recently shown that thin films of intermediate-length alkanes do not completely wet $\mathrm{SiO}_{2}$ surfaces. For C32 films they detect a narrow temperature range between 337 and $341 \mathrm{~K}$ in which a monolayer of perpendicular molecules spreads outward from a mesa-shaped particle. Above this temperature range, the monolayer delayers to form liquid droplets.

In previous studies using high resolution ellipsometry, Volkmann et al. ${ }^{16}$ performed heating-cooling cycles on C32 films grown by fast dip coating on $\mathrm{SiO}_{2}$-coated $\mathrm{Si}(100)$ substrates. Measurements of heating-cooling cycles on films with thicknesses ranging from $\sim 110 \AA$ down to $\sim 5 \AA$ show evidence of sharp steps in the ellipsometric signal below the bulk melting point at $341 \mathrm{~K}$. In low-coverage films they identified a single substep at $\sim 332 \mathrm{~K}$ which they suggested might correspond to a crystalline-to-rotator transition in a single layer of molecules oriented perpendicular to the surface. At higher coverage, they observed an additional substep at $\sim 336 \mathrm{~K}$ identified with the bulk crystalline- $R_{\mathrm{III}}$ phase transition described above. However, at that time no complementary topographical information from, e.g., scanning probe microscopy measurements was available and no films free of bulk particles had been grown to confirm the 
identification of the substep at lower temperature. Since then, no further work has been performed to study in detail the substeps in the ellipsometric signal.

Schollmeyer et al. ${ }^{13}$ conducted a comprehensive study of triacontane $\left(n-\mathrm{C}_{30} \mathrm{H}_{62}\right.$ or $\left.\mathrm{C} 30\right)$ films grown by spin coating on solid substrates. From their X-ray diffraction measurements, they found that some of their thin films had a different number of solid phases than the bulk material and suggested that these additional phases were stabilized by interfacial interactions. Due to the amount of material in their samples, bulk crystallites complicated the interpretation of transitions occurring in the monolayer and multilayer structures.

Other ellipsometric experiments have been performed to study phase transitions in alkane films. Ellipsometry and surface tension measurements ${ }^{17}$ show that at the liquid-alkane/ air interface the surface freezing effect can be detected as a sharp step corresponding to a change in the ellipticity coefficient $\boldsymbol{\rho}$, as well as by a change in the slope of the surface tension when plotted as a function of temperature. Pfohl et al. ${ }^{17}$ explain these changes in terms of a fluctuation of the molecules at the interface, which goes from a smectic-like order to a nematic-like order. Films consisting of a mixture of alkane and a surfactant show a similar behavior at the alkane-solution/air interface. ${ }^{18}$ In those studies, no changes in the ellipsometric signal have been observed below the bulk melting point.

In this work, we perform measurements with highresolution ellipsometry and atomic force microscopy (AFM) on C32 films with thicknesses ranging from $\sim 30$ to $\sim 150 \AA$ deposited on a solid substrate. Contrary to previous studies, we were able to grow thin films free of bulk particles. Due to the high sensitivity of our ellipsometer, we can identify phase transitions occurring below the bulk melting point. These phase transitions appear as abrupt steps in the ellipsometer signal as a function of temperature. In the thinnest films, we observe one step, which comes from the perpendicular monolayer without superposition of signals from bulk particles. In higher coverage samples, we detect in heatingcooling cycles a combination of features that we attribute to multilayer molecular films coexisting with bulk material.

Following the notation of Holzwarth et al. ${ }^{8}$ we can classify our films in terms of coverage. Films with ellipsometric thicknesses (defined in Sec. II B) less than the all-trans length of a C32 molecule $(42.5 \AA)^{5,11,16,19}$ will be denoted as "submonolayer coverage," i.e., the number of adsorbed molecules is insufficient to fill a complete perpendicular monolayer. Films with ellipsometric thicknesses greater than the all-trans length will be called "high coverage" or "excess coverage" films. The high-coverage samples that we investigate here have an almost complete first perpendicular monolayer, islands of second perpendicular layers, and almost no bulk particles. On the other hand, the excess-coverage samples have several perpendicular layers and a significant number of bulk particles.

\section{EXPERIMENTAL METHODS}

\section{A. Materials}

The samples consist of thin films of C32 deposited on $\mathrm{Si}(100)$ substrates with their native oxide layer. The C32
TABLE I. Experimental values used for calculations with the Drude model, the thin film approximation (TFA), and the Clausius-Mossotti (CM) relation.

\begin{tabular}{lc}
\hline \hline Parameter & Value \\
\hline Angle of incidence $\varphi(\mathrm{deg})$ & 60.5 \\
Laser wavelength $\lambda_{0}(\AA)$ & 6328 \\
Substrate refraction index & $n=3.877+i 0.019$ \\
Substrate dielectric constant & $\varepsilon_{s}=15.03+i 0.14$ \\
(Initial) film refraction index & $n=1.459$ \\
(Initial) film dielectric constant & $\varepsilon_{b}=2.129$ \\
Lattice constants $a, b, c(\AA)$ & $7.57,4.98,42.5$ \\
\hline \hline
\end{tabular}

(Aldrich) had a purity of $99.9 \%$ and was used as supplied. The polished silicon wafers (Virginia Semiconductor Inc.) were cut into pieces of about $11 \times 11 \mathrm{~mm}^{2}$ and were cleaned using a solution of sulfuric acid and hydrogen peroxide described in more detail in Ref. 20. This cleaning procedure does not remove the native oxide layer and gives very reproducible substrates. ${ }^{20,21}$ We consider a clean substrate to be any silicon wafer that yields a polarizer angle $P=43.90^{\circ}$ at a preset (fixed) analyzer angle $A=23.2560^{\circ}$. These angles are valid for a null-ellipsometer setup with the incident angle and wavelength given in Table I. ${ }^{22}$

We used two different methods to grow the films: (a) slow dip coating (SDC) at room temperature in a C32/ heptane solution and (b) physical vapor deposition (PVD). The SDC method is described elsewhere. ${ }^{23}$ With this simple technique, we obtained submonolayer films having morphology very similar to those obtained by spin coating but without coexisting bulk particles. ${ }^{8,24}$ To determine the ellipsometric thickness of each SDC film, we measured the absolute $P$ angle and compared it with the absolute $P_{0}$ angle for the clean substrate as described in the next section.

The PVD process was performed in high vacuum $\left(\sim 10^{-6}\right.$ Torr $)$ with the substrate held at $314 \mathrm{~K}$. This substrate temperature and the deposition rates have been determined experimentally in order to obtain smoother films. Higher substrate temperatures led to desorption of the film in high vacuum. During deposition, the ellipsometric thickness was continuously monitored until reaching the desired thickness. After deposition, the films were annealed in high vacuum for about $5 \mathrm{~h}$ with the substrate held at $314 \mathrm{~K}$. By comparing AFM images of these samples before and after annealing in high vacuum, we concluded that the major effect of annealing was to desorb bulk material that nucleated during PVD. ${ }^{12}$

\section{B. Ellipsometry}

We used a system composed of a custom-made ellipsometer coupled to a high-vacuum chamber equipped with a Knudsen cell. This high-vacuum high-resolution ellipsometer (HV-HRE) system allows us to grow films by PVD and to perform temperature cycles while monitoring in situ and in real time the changes in the polarization angle. The ellipsometer setup has been described previously. ${ }^{16,22}$ Briefly, we use a null-PCSA configuration ${ }^{25}$ with a fixed wavelength $\lambda_{0}$ and fixed angle of incidence $\varphi$, whose values are given in Table I. The resolution of the apparatus is of the order of $10^{-3} \mathrm{deg}$ in 
the polarizer angle, which is achieved by using a Faraday glass placed between the polarizer and the compensator and controlled by an electronic feedback loop.

When plane polarized laser light passes through a layer of alkane molecules and is reflected from the substrate, the angle of polarization of the laser beam changes. The change $d P$ in the polarization angle may be expressed in terms of the alkane film thickness $l$ and the optical properties of this film and substrate: ${ }^{26-28}$

$$
d P=\Gamma\left(\varepsilon_{t}, \varepsilon_{n}, \varepsilon_{s}\right) l,
$$

with

$$
\Gamma\left(\varepsilon_{t}, \varepsilon_{n}, \varepsilon_{s}\right)=\frac{2 \pi}{\lambda_{0}} \cos \varphi\left(\frac{1}{1-\varepsilon_{s}}\right) \frac{\varepsilon_{t}-\varepsilon_{s}-1+\varepsilon_{s} / \varepsilon_{n}}{\cot ^{2} \varphi-1 / \varepsilon_{s}} \frac{180}{\pi} .
$$

Here the adsorbed film is characterized by a tangential (parallel to the substrate) dielectric constant $\varepsilon_{t}$ and a normal (perpendicular to the substrate) dielectric constant $\varepsilon_{n}$, whereas the substrate is assumed to behave as an isotropic optical medium with complex dielectric constant $\varepsilon_{s}$. The other parameters are defined in the previous paragraph. In our calculations, we consider an isotropic dielectric constant for the film $\left(\varepsilon_{t}=\varepsilon_{n}=\varepsilon_{\text {bulk }} \equiv \varepsilon\right)$. We can solve Eq. (2) numerically for the values listed in Table I, assuming a thin film approximation (TFA), where $l \ll \lambda_{0}$. Replacing in Eq. (1) we find

$$
d P=0.05198 l
$$

where the change in polarization angle $d P$ is expressed in degrees and both the film thickness $l$ and the laser wavelength $\lambda_{0}$ are in angstroms. We solved the complete Drude equations using a FORTRAN routine, which has been tested in previous studies. $^{29,22}$

In Eq. (1), it was assumed that the film thickness is uniform in the entire area illuminated by the laser beam. For films with nonuniform thickness, the reflected beam is a superposition of beams with different changes in the polarization angle, so the measured $d P$ represents an average thickness of the film, which we called "ellipsometric thickness." If the film has the same thickness over the entire illuminated area, the ellipsometric thickness is equal to the geometrical thickness. To obtain better insight into the distribution of molecules on the substrate, we use amplitude modulation AFM to scan over several distant zones. The scanner of our AFM has a maximum range of nearly $50 \times 50 \mu \mathrm{m}^{2}$ and is mounted on a precision translational stage. Since the area illuminated by the laser beam is usually larger than the area scanned by AFM, we select areas separated by several micrometers in our AFM measurements.

Equation (3) can be used to determine ellipsometric film thicknesses from a measured change in the polarization angle, as long as the optical properties of the molecules do not change. However, if a film of given ellipsometric thickness is studied as a function of temperature, changes in the conformation of the constituent molecules may occur that may change the dielectric constant. In that case, small changes in $d P$ can be observed with a high-resolution ellip- someter, even if the film coverage does not change, since $\Gamma$ in Eq. (1) changes. In this case, it would be useful to express the dielectric constant of the film as a function of the volumetric density, using the Clausius-Mossotti (CM) relation: ${ }^{30}$

$$
\frac{\varepsilon_{r}-1}{\varepsilon_{r}+2}=\frac{\rho N_{A v o} \alpha}{3 M \varepsilon_{0}},
$$

where $\rho N_{A v o} / M$ is the number density of molecules with polarizability $\alpha$, and $\varepsilon_{r}, \varepsilon_{0}$ are the relative and vacuum permittivity, respectively.

Another ellipsometric model for thin films was proposed by Dignam and Fedyk, ${ }^{31}$ in which the molecules in the film are assumed to be orientationally ordered such that dipoles are induced in the molecules by the external electromagnetic field of the laser light. In this approximation Dignam and Fedyk used the relations between the molecular polarization $\alpha$ and the film surface susceptibilities resulting from the interaction:

$$
\gamma=\gamma(\alpha, N),
$$

where $N$ is the number density of molecules per unit area. They obtained an equivalent relation to Eq. (1), in terms of the susceptibilities and change in polarization $d P$ :

$$
d P=\frac{2 \pi}{\lambda_{0}} \frac{\cos \varphi}{1-\varepsilon_{s}} \frac{\Delta \gamma_{t}-\varepsilon_{s} \Delta \gamma_{n}}{\cot ^{2} \varphi-1 / \varepsilon_{s}} \frac{180}{\pi},
$$

where $\Delta \gamma_{j=t, n}$ denotes changes in the normal and tangential components of the susceptibility.

\section{Temperature cycles}

The sample holder in the HV-HRE system is equipped with a heater and a Pt resistance thermometer connected to a temperature controller, which allows us to perform linear ramps (heating or cooling) with a resolution of $0.1 \mathrm{~K}$. Temperature cycles were performed in air at atmospheric pressure at a rate of $0.5 \mathrm{~K} / \mathrm{min}$, beginning at room temperature. For some samples, we ramped the temperature well above the bulk surface freezing transition $T_{s}(345 \mathrm{~K})$ to reach the liquid phase. In other samples, we ended the heating only a few degrees above the first observed step in $d P$ and then cooled down in order to study the hysteresis of the transition. Between temperature cycles, the topography of some samples has been analyzed with AFM to monitor changes. Also, some thermal cycles were performed at a different temperature ramp rate to investigate possible hysteresis effects.

\section{RESULTS}

We studied five samples in each of three different thickness ranges: Submonolayer coverage $(l<45 \AA)$, high coverage $(l \sim 50 \AA)$, and excess coverage $(l>90 \AA)$. All of our samples were stable over a period of weeks when stored at room temperature in air. For samples of submonolayer coverage, a single step in $d P$ of nearly constant magnitude was observed in successive heating-cooling cycles. However, in high-coverage and excess-coverage samples several steps in $d P$ could be observed initially, but their number decreased in subsequent heating-cooling cycles. Below, we show representative results for each type of sample. 
a

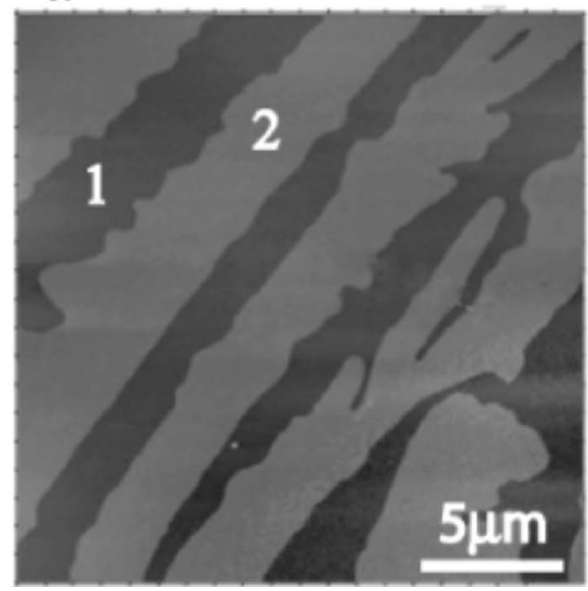

b

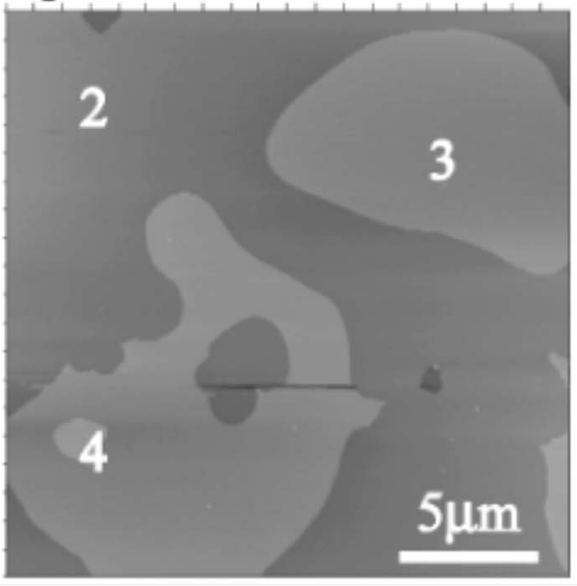

C

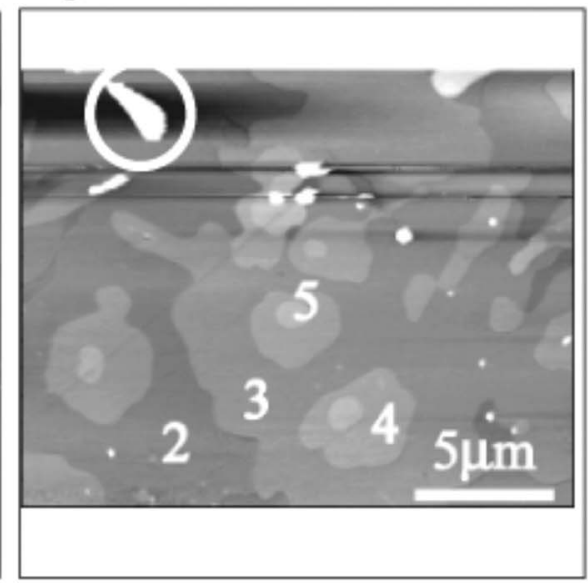

FIG. 1. Topographic AFM images of scan areas $20 \times 20 \mu \mathrm{m}^{2}$ taken in the amplitude modulation AFM mode on C32 samples of three different coverages: (a) a submonolayer SDC sample of ellipsometric thickness $\sim 35 \AA$. Stripes of molecules (labeled 2) oriented with their long axis perpendicular to the SiO 2 surface reside on a complete bilayer of molecules (labeled 1) oriented with their long axis parallel to the surface. (b) A high-coverage sample of ellipsometric thickness $\sim 50 \AA$ A. The first perpendicular layer (labeled 2) covers almost the entire scan area; above it are islands that contain a second (labeled 3) and third (labeled 4) perpendicular layer. (c) An excess-coverage PVD sample of ellipsometric thickness $\sim 90 \AA$ A; more islands of multiple perpendicular layers appear as well as bulk particles (white circle). A large bulk particle in the upper area of the image causes noise; for this reason, we deleted that part of the image to achieve better contrast.

In the submonolayer regime, we prepared samples by both, SDC and PVD. Figure 1(a) shows a topographic AFM image taken in the amplitude modulation mode of a submonolayer film of thickness $\sim 35 \AA$ prepared by SDC. It shows a perpendicular monolayer with a striped morphology without bulk material present. The stripes are aligned in the direction in which the sample was pulled from the solution. In contrast, submonolayer samples prepared by PVD exhibit fractal-like islands of the perpendicular monolayer (not shown), which are characteristic of diffusion-limited aggregation. ${ }^{24,32}$

The high-coverage films have an ellipsometric thickness of $\sim 50 \AA$. AFM images [see Fig. 1(b)] reveal that this type of sample consists of an incomplete first perpendicular layer and islands of a second perpendicular layer. From Fig. 1(b) we calculated an average geometrical thickness of $\sim 70 \AA$, somewhat higher than the ellipsometric thickness. As noted above, the ellipsometric thickness represents an average over the illuminated area, which is approximately 2500 times larger than the scan area of the AFM images in Fig. 1.

Figure 1(c) shows a topographic AFM image of a PVD film with an ellipsometric thickness of $\sim 90 \AA$, corresponding to an excess-coverage sample. For these samples we observe islands containing up to four perpendicular layers, while in dip-coated samples of the same ellipsometric thickness we find bulk particles instead of the layered structure.

We measured the temperature dependence of the ellipsometer signal in heating-cooling cycles on each sample at temperatures from $\sim 300 \mathrm{~K}$ up to $350 \mathrm{~K}$ at rates of 0.5 $\mathrm{K} / \mathrm{min}$. Figure 2 shows plots of $d P$ measured during the heating part of the cycle for each kind of sample. In Fig. 2(a), we plotted $d P$ as a function of temperature for a submonolayer sample. A sharp step in $d P$ of $\sim 0.04^{\circ}$ is observed at $330.5 \mathrm{~K}$. When heating-cooling cycles were performed at a different rate, from 0.5 to $2 \mathrm{~K} / \mathrm{min}$ (not shown), we observed a hysteresis of $\sim 1.6 \mathrm{~K}$, which is independent of the ramp rate. Between the first and the second cycle, the temperature of the step shifts downward by $\sim 0.5 \mathrm{~K}$ as shown in Fig. 3. The uncertainty in the determination of the transition temperature for these samples is $\pm 0.5 \mathrm{~K}$. After a few more heating cycles, the topography of these samples tends to change from
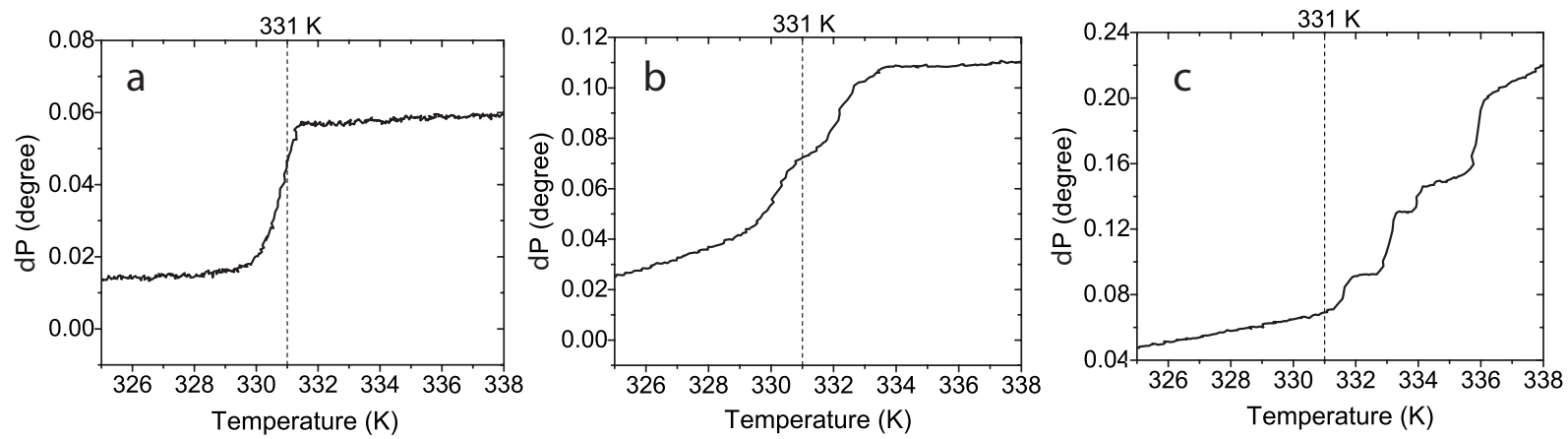

FIG. 2. Changes in the ellipsometric angle $P$ as a function of temperature for the three samples in Fig. 1 . The resolution of $0.001^{\circ}$ is of the order of the line thickness in the graphs. (a) The submonolayer coverage sample shows a step height of $0.04^{\circ}$ at $\sim 331 \mathrm{~K}$, (b) the high-coverage PVD sample has two "distorted" steps, and (c) the excess-coverage PVD sample shows four steps, the last coinciding with the rotator transition for bulk C32. 


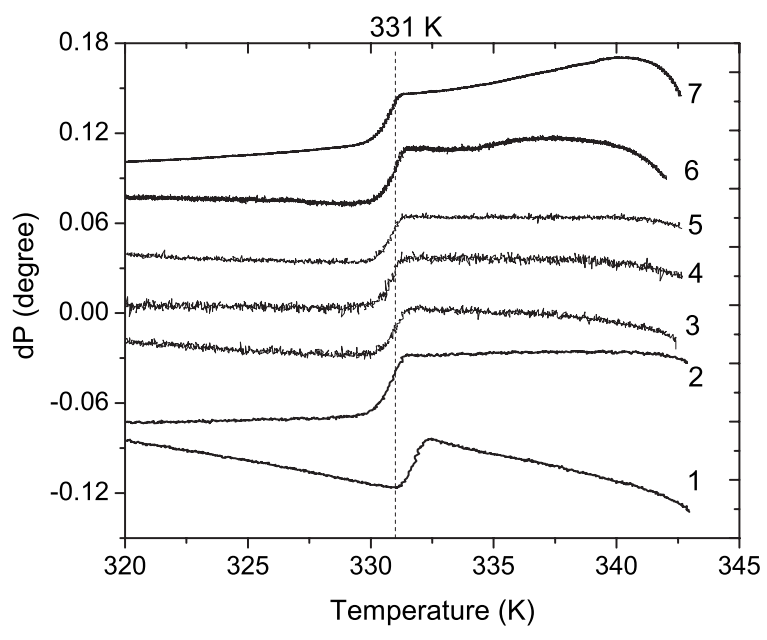

FIG. 3. Ellipsometric signal measured in successive heating cycles from the submonolayer SDC sample in Figs. 1(a) and 2(a). Numbers at the right indicate the cycle number. Successive temperature scans have been displaced vertically for clarity. These scans show two effects: The first temperature scan always has a different shape (negative slope of the background signal) and is shifted slightly up in temperature with respect to the others. Also, the upper region of the curves changes slightly as the number of cycles increases. We performed AFM measurements after each temperature cycle and observed the stripes [see Fig. 1(a)] develop into fractal domains. The observed change in the slope of the background signal is characteristic for SDC samples and related to the loss of the striped structure. The rounding of $d P$ in cycles 6 and 7 at temperatures above $340 \mathrm{~K}$ may be a precursor of delayering (Ref. 4).

striped to seaweed-like structures similar to ones shown in Ref. 23.

Despite differences in the submonolayer film morphology at room temperature, films grown by SDC and PVD both showed a single step up in $d P$ at $\sim 331 \mathrm{~K}$ on heating as illustrated for an SDC sample in Fig. 3. Thus, we find that the temperature dependence of the observed ellipsometric signal is independent of the growth method and morphology of the first perpendicular layer.

AFM images of our submonolayer samples did not show evidence of delayering or formation of mesa-shaped particles after at least eight heating-cooling cycles to temperatures above $T_{b}=341 \mathrm{~K}$ in contrast with high-coverage and excesscoverage samples. ${ }^{4}$

Figure 2(b) shows $d P$ as a function of the temperature for a high-coverage sample with an ellipsometric thickness of $\sim 50 \AA$. The behavior of these samples is not as reproducible as that of the submonolayer and excess-coverage samples. For the sample in Fig. 2(b), we observe two steps in $d P$ on its first heating cycle, at $\sim 331$ and $\sim 333 \mathrm{~K}$. The height of both steps is $\sim 0.03^{\circ}$ and they are less sharp than in the case of the submonolayer sample. For subsequent heating-cooling cycles of this sample, only a single step can be identified at $332 \mathrm{~K}$, somewhat above the single step in the submonolayer film of Fig. 2(a). However, another highcoverage film showed two steps in the first heating-cooling cycle at 333 and $337 \mathrm{~K}$, which remain unchanged in subsequent cycles. Thus, based on the number of steps observed in $d P$, our high-coverage samples behaved either like a submonolayer or as an excess-coverage sample, as we will discuss below.

For an excess-coverage sample, we identify three steps

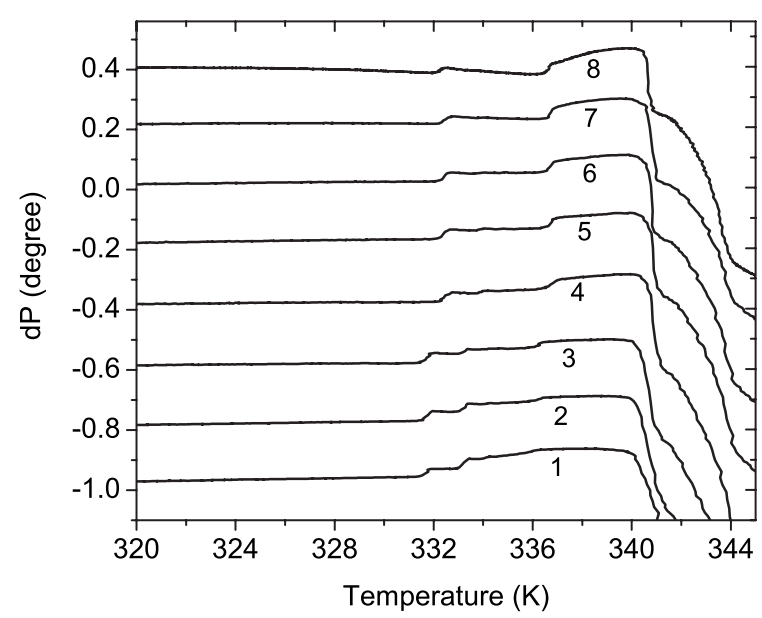

FIG. 4. A series of heating cycles on an excess coverage PVD sample. Successive temperature scans have been displaced vertically for clarity. The lowest curve corresponds to the first cycle and the upper curve (eighth) to the last cycle. We observe that in the first three cycles the steps are shifted to slightly lower temperature values. For cycles 1-5 we detect three steps and for cycles 6-8 there are only two steps.

in the ellipsometer signal: At $\sim 332, \sim 333$, and $\sim 336 \mathrm{~K}$ on its first heating [Fig. 2(c)]. In Fig. 4, we show a series of heating cycles on another excess-coverage sample. This sequence shows the step at $\sim 333 \mathrm{~K}$ disappearing and only the last two steps at $\sim 332$ and $\sim 336 \mathrm{~K}$ remaining in the last three cycles. The temperatures of these two steps are in good agreement with the two steps found earlier by Volkmann et $a l .{ }^{16}$ for dip-coated samples of comparable thickness. The highest-temperature step is close to that reported for the bulk C32 crystalline-to-rotator transition. ${ }^{1}$ We also prepared new dip-coated samples with ellipsometric thickness of $\sim 100 \AA$, which gave similar results.

\section{DISCUSSION}

Comparing the AFM topographies in Fig. 1 with the ellipsometric signals in Fig. 2, we find that as the number of partly occupied islands of perpendicular layers increases, the number of steps in the ellipsometer signal $d P$ also increases. This correlation suggested to us that a step in $d P$ might be caused by a phase transition occurring in the multilayer islands of perpendicular molecules. The highest-temperature step at $\sim 337 \mathrm{~K}$ appears to coincide with the bulk C32 crystalline- $R_{\mathrm{III}}$ phase transition ${ }^{1,5}$ and the lower step at $\sim 332 \mathrm{~K}$ can be correlated with a solid-solid phase transition in the multilayer islands of perpendicular molecules. Previous synchrotron x-ray diffraction measurements of Bai et al. ${ }^{4}$ provide further experimental evidence of a structural phase transition below the bulk crystalline- $R_{\text {III }}$ phase transition in C32 films adsorbed on a $\mathrm{SiO}_{2}$ surface. They interpreted a change in the in-plane diffraction patterns at $329-330 \mathrm{~K}$ on heating as resulting from a phase transition from an orthorhombic to a hexagonal structure in mesa-shaped C32 particles of thickness ranging up to a few lengths of a molecule.

We propose that the disappearance of the step in $d P$ at $\sim 333 \mathrm{~K}$ that occurs between cycles 5 and 6 in Fig. 4 results from a diffusion of molecules from the multilayer islands. In high and excess coverage samples, molecules diffuse during 
heating-cooling cycles from the multilayer islands to bulk particles, leaving a very low occupancy of upper perpendicular layers. A concomitant increase in size of the coexisting bulk C32 particles appears to be consistent with the increased step height in $d P$ at $\sim 337 \mathrm{~K}$ attributed to the crystalline- $R_{\text {III }}$ phase transition as well as a sharper downward step at $\sim 341 \mathrm{~K}$, the bulk C32 melting point. We associate the remaining step at $\sim 332 \mathrm{~K}$ with the crystalline to plastic transition of the multilayer islands. Due to the large occupancy of the perpendicular monolayer, this step remains after several thermal cycles. These results show that the perpendicular layers are not stable through heating-cooling cycles in ambient conditions and they tend to form bulk particles or three-dimensional mesa-shaped particles.

In the remainder of this section, we focus on the origin of the single step in $d P$ observed at $\sim 331 \mathrm{~K}$ in the submonolayer films [Figs. 2(a) and 3]. From the AFM image in Fig. 1(a) and from other submonolayer samples, we can be reasonably confident of the absence of bulk particles and multilayer islands of perpendicular molecules. This conclusion is also supported by the absence in the ellipsometric scans of a step in $d P$ at $\sim 337 \mathrm{~K}$, the temperature of the bulk C32 crystalline- $R_{\mathrm{III}}$ phase transition. Therefore, we wish to consider what type of solid-solid phase transition in a perpendicular monolayer can contribute a single step upward in $d P$ on heating.

We suggest that the change in the background level observed in Fig. 3 in the series of heating cycles is unrelated to the transition at $\sim 331 \mathrm{~K}$. The background signal is related to changes in the morphology of the perpendicular monolayer of $\mathrm{C} 32$ molecules during the heating-cooling cycles. From AFM images taken after each heating cycle (not shown), we know that the partial perpendicular monolayer evolves from a nearly perfect striped structure to "stripes with fingers" and finally to seaweed-like structures. In general, such changes in the morphology of a layer, occurring on a length scale of the wavelength of the ellipsometer's laser light or larger, can affect the background level in the measurement of $d P$. The rounding of $d P$ in cycles 6 and 7 in Fig. 3 at temperatures above $340 \mathrm{~K}$ may be a precursor of delayering. ${ }^{4}$ However, the mentioned absence of a step at $\sim 337 \mathrm{~K}$ in our submonolayer coverage samples is an additional indicator for a nearly bulk particle free perpendicular C32 layer. This is an important difference with respect to the excess coverage sample (Fig. 4).

Recent synchrotron x-ray reflectivity (XRR) measurements on high-coverage PVD films of C32 indicated that the thickness of the first perpendicular layer decreases from the all-trans length of the molecule $42.5 \AA$ (Ref. 12) at room temperature to $40.3 \AA$ at $338 \mathrm{~K}$. A possible explanation for this reduction in layer thickness on heating may be a decrease in the length of the molecules caused by creation of gauche defects within them. ${ }^{33,34}$ Extending this view, one might consider a phase transition in which gauche defects are introduced collectively into the molecules such as in a "chain-melting" transition. ${ }^{33}$ In this way, we were led to the possibility that the substep in $d P$ at $\sim 331 \mathrm{~K}$ might result from a solid-solid phase transition in which gauche defects are collectively introduced into the alkane chains. One
TABLE II. Summary of results from the two model calculations for thin films. The column "CM+DRUDE" corresponds to the application of the $\mathrm{CM}$ relation [Eq. (4)] in combination with the complete Drude model, while the column "CM+TFA" corresponds to the use of the CM relation [Eq. (4)] in combination with the TFA, as summarized in Eqs. (1)-(3).

\begin{tabular}{lcc}
\hline \hline Results & CM+DRUDE & CM+TFA \\
\hline Final film refraction index $n$ & 1.481 & 1.478 \\
Final film dielectric constant $\varepsilon_{b}$ & 2.193 & 2.184 \\
Final film thickness $(\AA)$ & 40.9 & 41.06 \\
\hline \hline
\end{tabular}

gauche defect reduces the length of the chain by $\sim 1.3 \AA$. We estimate that one or two defects per molecule on average would be sufficient to explain the $\sim 5 \%$ decrease in monolayer thickness.

To test this hypothesis, we use the TFA and the complete Drude formalism, both in combination with the CM relation. In the case of the TFA, we start with Eqs. (2) and calculate the change in film dielectric constant $\varepsilon$ for a change in polarizer angle of $0.04^{\circ}$. Then we use Eq. (4) to calculate the change in volume density, which corresponds to the calculated change in film dielectric constant $\varepsilon$. Because there is no independent experimental evidence for a change in the monolayer lattice constant at $\sim 331 \mathrm{~K}$, we can translate the resulting $3.5 \%$ change in volume density into a shrinking from the initial monolayer thickness of $42.5 \AA$ to a final thickness of $41.06 \AA$. We check this hypothesis applying the complete Drude formalism: First we used Eq. (4) to calculate the change in film dielectric constant from a supposed change in volume density. Then we evaluate the calculated change in dielectric constant, using the complete Drude equations to calculate the corresponding change in $d P$. With this procedure we reproduce the measured step up of $0.04^{\circ}$ for an increase of $4 \%$ in volumetric density. This corresponds to a decrease in film thickness from the initial $42.5 \AA$ to 40.9 $\AA$. As we expected, both methods lead to very similar numerical results for the proposed shrinking in monolayer thickness. The results of both procedures are summarized in Table II, while the parameters used for the calculations are summarized in Table I.

From a theoretical point of view, the step height should depend on the coverage and in principle we could introduce a coverage related correction factor for $d P$. For the range of monolayer occupancy reported in this work, from $\sim 50 \%$ to $\sim 70 \%$, we observe the same step height of $\sim 0.04^{\circ}$ in $d P$. If we consider a linear scaling between the measured $d P$ step height and a $100 \%$ monolayer coverage, we expect for the hypothetical complete coverage a $d P$ of $<0.06^{\circ}$. Using $\mathrm{CM}+\mathrm{TFA}$ for this case, we calculate a shrinking from the initial $42.5 \AA$ to a final thickness of $\sim 40.5 \AA$. This value is very close to the shrinking of $40.3 \AA$ observed with XRR. ${ }^{12}$ Considering the almost constant step height we measured at monolayer coverages of $\sim 50 \%$ and $\sim 70 \%$, a linear scaling may be regarded as the upper limit for a $d P$ correction.

We also considered the case if the in-plane lattice constant were to increase so that the covered fraction would increase with the total number of adsorbed molecules remaining constant. In this case, we obtain a decrease in film refractive index (and dielectric constant), which would lead 
in our ellipsometer setup to a step down in $d P$, opposite to the observed effect. In addition we considered the case if for some reason molecules in the first layer of a submonolayer film are promoted into a second layer. In this case, we do not expect an effect on the polarizer angle and no change in $d P$. In an ellipsometry measurement it is therefore not possible to distinguish between these two structures.

A second scenario to explain the measured step up in the ellipsometric signal could arise from an "untilt" of initially tilted molecules. We analyze this case with the Drude model, keeping the refractive index of the film constant and interpret the step up of $0.04^{\circ}$ as caused by an increase in film thickness of $0.74 \AA$. Such an increase in thickness requires the molecules of the first "perpendicular" monolayer to be slightly tilted at $\sim 10.7^{\circ}$, for temperatures below $331 \mathrm{~K}$. At the phase transition the molecules collectively stand up. However, there is no experimental evidence for an increase in film thickness. Because of the small increase in thickness required to explain the step up with the "untilt" hypothesis it could be argued that the complementary techniques of x-ray reflectivity and AFM measurements do not have sufficient precision to observe such a small tilting effect and the related $0.74 \AA$ thickness increase in the perpendicular monolayer. $^{4,14,15,23}$ Going one step further, we have to assume that the supposed tilted monolayer is stabilized for $T<331 \mathrm{~K}$ by some mechanism. An obvious argument for stabilization considers the molecules as snapped-in to their zig-zag conformation. At the transition temperature, the molecules spontaneously snap out of this configuration, aligned perpendicular to the substrate. However, such conformational changes require an increase in the nearest-neighbor distance or decrease in number density, which according to Eq. (2) would cause a step down in the ellipsometric $d P$ signal. Then the observed step up of $0.04^{\circ}$ would be the net effect of a step up (from the supposed "untilt" of the molecules) and a step down (from the supposed increase in lattice constant). In this hypothetical case, the contribution from an "untilt" of the molecules must be larger than calculated above. The initial tilt angle must be $>10.7^{\circ}$ and the expected increase in film thickness must exceed $0.71 \AA$, reaching the range accessible with diffraction techniques and AFM measurements.

In addition, we explored the origin of the step up as a result of a change in the microscopic parameter of molecular polarizability. For this purpose, we used approximations based on the Dignam and Fedyk (D\&F) model. ${ }^{31}$ In the case of submonolayer films, we can expect that the bulk dielectric permittivity is not an ideal optical parameter to model the interaction with light. In the microscopic model proposed by Dignam and Fedyk, the film is adsorbed on an isotropic substrate and is considered to be a homogeneous twodimensional array of dipoles induced by an external electrostatic field and by the field originating from the induced dipoles in the other molecules. In our approximations, we only vary the molecular polarizability and maintain the number density and lattice parameter constant, because x-ray experiments ${ }^{11,4}$ do not indicate considerable changes in both latter.

We assume that the molecular crystal arrangement has a
TABLE III. Approximative comparison of the normal polarizability $\alpha_{n}$ and the mean tangential polarizability $\alpha_{t}$ before and after the observed phase transition, based on the D\&F model (Ref. 31). The tangential polarizability almost does not change due the transition, but the normal polarizability decreases approximately $22 \%$ [calculated with $G(0)=7.255$ ] to $35.5 \%$ [calculated with $G(0)=11.034]$.

\begin{tabular}{lcccc}
\hline \hline D\&F approximations & $\begin{array}{c}\alpha_{n} \\
\left(\AA^{3}\right)\end{array}$ & $\begin{array}{c}\alpha_{t} \\
\left(\AA^{3}\right)\end{array}$ & $\begin{array}{c}\Delta \alpha=\alpha_{n}-\alpha_{t} \\
\left(\AA^{3}\right)\end{array}$ & $\begin{array}{c}\text { Change } \alpha_{n} \\
(\%)\end{array}$ \\
\hline Before transition & 43.96 & 36.49 & 7.47 & \\
After transition $[G(0) \sim 7.255]$ & 34.15 & 36.28 & -2.13 & $\sim 22$ \\
After transition $[G(0) \sim 11.034]$ & 27.93 & 34.37 & -6.44 & $\sim 35.5$ \\
\hline \hline
\end{tabular}

hexagonal structure with a lattice constant of $a=4.67 \AA$ that corresponds to the number density reported by Bai et al. ${ }^{4} \mathrm{We}$ also assume that the molecules are in their all-trans configuration. From semiempirical quantum calculations at the AM1 level ${ }^{35}$ we obtain initial values for the normal and tangential component of $\mathrm{C} 32$ polarizability, $\alpha_{n}$ and $\alpha_{t}$, respectively, from which we can approximate with the D\&F model ${ }^{31}$ the corresponding values $\alpha_{n}^{\prime}$ and $\alpha_{t}^{\prime}$ after the phase transition. The results, summarized in Table III, suggest that the anisotropy in the polarizability of the $\mathrm{C} 32$ molecules almost disappears at the transition. To produce the observed step of $0.04^{\circ}$ in $d P$ we calculated with the D\&F model changes in the normal polarizability of $\sim 22 \%$ to $\sim 35 \%$, for two different geometrical factors $G(0),{ }^{31}$ a crude integral approximation for the dipole sum with $G(0) \sim 7.255$ and a numerical result for an hexagonal net with $G(0) \sim 11.034$, respectively. In $G(h)$ in the D\&F model, the parameter $h$ is $h=z / a$, where $z$ is the distance from the molecules in the array to the dipole images they form on the substrate, and $a$ is the lattice parameter. In our case $h$ is $\sim 9$ and for large $h$ the leading powers cancel between the two terms and $G(h) \sim-3 / h^{5}$ (Refs. 31) and therefore this image sum is completely negligible.

The resulting changes in molecular polarizability can be related to a combination of defects in the all-trans configuration of the $\mathrm{C} 32$ molecules, such as gauche defects. ${ }^{36} \mathrm{We}$ used semiempirical quantum calculations at the AM1 level ${ }^{35}$ to check if the calculated changes in the polarizabilities could be caused by the introduction of gauche defects in the molecules. We found that the changes in the polarizability were less than $1 \%$ with up to four defects in the molecule. A formation of up to four gauche defects is already beyond the limit of consistency with observed changes in film thickness. ${ }^{12}$ For this reason, we also discard this third possibility of a collective change in molecular polarizability to explain the $d P$ step of $\sim 0.04^{\circ}$ and retain the first scenario discussed (compression of the film thickness with the corresponding increase in the film refractive index probably due to the formation of one to two gauche defects).

\section{SUMMARY}

We observed sharp steps in the ellipsometric signal from C32 films adsorbed on $\mathrm{SiO}_{2}$-coated $\mathrm{Si}$ surfaces, below the bulk melting point. These features have been reported before for higher coverage films but no systematic ellipsometric measurements have been performed to study their stability and reproducibility under thermal cycling. By investigating 
the temperature dependence of the ellipsometric signal $d P$ at progressively higher $\mathrm{C} 32$ coverages, we find that the number of steps in the signal increases with the number of partly occupied layers of molecules. After thermally cycling our high-coverage samples above the bulk $\mathrm{C} 32$ melting point, we observe two steps in $d P$ at temperatures of $\sim 332$ and $\sim 337 \mathrm{~K}$, in agreement with previous measurements. ${ }^{16}$ The step at $\sim 337 \mathrm{~K}$ corresponds to the bulk crystalline- $R_{\mathrm{III}}$ phase transition. We suggest that the step at $\sim 332 \mathrm{~K}$ is associated with a solid-solid transition in multilayer islands of perpendicular molecules.

In addition, we investigated for the first time the ellipsometric signal from submonolayer samples without superposition of signals from multilayer islands or bulk particles. In these samples only one sharp step is observed at $\sim 331 \mathrm{~K}$ with a hysteresis of about $\sim 1.6 \mathrm{~K}$ between heating and cooling. We have investigated three possible explanations for this step up in the ellipsometric signal $d P$. Based on recent synchrotron $\mathrm{X}$-ray reflectivity measurements on similar films, ${ }^{12}$ we proposed that this step is due to a "chainmelting" transition in which gauche defects are introduced collectively into the molecules. This transition produces a slight increase in the density within the monolayer islands, which is observed as a step up in the ellipsometric signal. The required density change corresponds to an average of about one or two gauche defects per molecule. We suggested a second explanation using the Drude model, based on molecules which were initially tilted with an angle of $\sim 10.7^{\circ}$, and then "untilt" at $\sim 331 \mathrm{~K}$ to a perpendicular orientation. The change in thickness required by such an explanation is opposed to that observed in XRR measurements on the same system. Finally, we considered that the step in the ellipsometric signal could be caused by a change in the molecular polarizability of C32. However, we discard this possibility, because the required change in molecular polarizability for a step up of $d P \sim 0.04^{\circ}$ is at least an order of magnitude larger than calculated for the formation of up to four gauche defects in the $\mathrm{C} 32$ chain.

Therefore, we strongly favor the first scenario in which the perpendicular monolayer decreases in thickness due to the collective formation of one or two gauche defects on average per molecule. This mechanism proposed for the generation of the step up in the first monolayer can also explain the additional steps observed in the samples with bilayer and trilayer islands at temperatures below the bulk crystalline- $R_{\mathrm{III}}$ phase transition at $T \sim 337 \mathrm{~K}$.

\section{ACKNOWLEDGMENTS}

The authors want to thank Professor Klaus Knorr for fruitful discussion. This work was supported by the Chilean government through FONDECYT Grant Nos. 1060628 and 7080105 and by CONICYT scholarships (E.A.C., V.d.C. and P.A.S.), and by the U.S. NSF Grant No. DMR-0705974.
${ }^{1}$ E. B. Sirota, H. King, Jr., H. Singer, and H. Shao, J. Chem. Phys. 98, 5809 (1993).

${ }^{2}$ B. Ocko, X. Wu, E. Sirota, S. Sinha, O. Gang, and M. Deutsch, Phys. Rev. E 55, 3164 (1997).

${ }^{3}$ A. Tkachenko and Y. Rabin, Phys. Rev. E 55, 778 (1997).

${ }^{4}$ M. Bai, K. Knorr, M. J. Simpson, S. Trogisch, H. Taub, S. N. Ehrlich, H. Mo, U. G. Volkmann, and F. Y. Hansen, Europhys. Lett. 79, 26003 (2007).

${ }^{5}$ M. Dirand, M. Bourouka, A.-J. Briard, V. Chevallier, D. Petitjean, and J. P. Corriou, J. Chem. Thermodyn. 34, 1255 (2002).

${ }^{6}$ K. Tozaki, H. Inaba, H. Hayashi, C. Quan, N. Nemoto, and T. Kimura, Thermochim. Acta 397, 155 (2003).

${ }^{7}$ C. Merkl, T. Pfohl, and H. Riegler, Phys. Rev. Lett. 79, 4625 (1997).

${ }^{8}$ A. Holzwarth, S. Leporatti, and H. Riegler, Europhys. Lett. 52, 653 (2000)

${ }^{9}$ K. Nozaki, R. Saihara, K. Ishikawa, and T. Yamamoto, Jpn. J. Appl. Phys., Part 1 46, 761 (2007).

${ }^{10}$ N. Maeda, M. Kohonen, and H. Christenson, Phys. Rev. E 61, 7239 (2000).

${ }^{11}$ H. Mo, S. Trogisch, H. Taub, S. N. Ehrlich, U. G. Volkmann, F. Y. Hansen, and M. Pino, J. Phys.: Condens. Matter 16, S2905 (2004).

${ }^{12}$ V. del Campo, E. Cisternas, H. Taub, I. Vergara, T. Corrales, P. Soza, U. G. Volkmann, M. Bai, S.-K. Wang, F. Y. Hansen, H. Mo, and S. N. Ehrlich, "Structure and growth of vapor-deposited $n$-dotriacontane films studied by x-ray reflectivity," Langmuir (in press).

${ }^{13}$ H. Schollmeyer, B. Struth, and H. Riegler, Langmuir 19, 5042 (2003).

${ }^{14}$ H. Mo, H. Taub, U. G. Volkmann, M. Pino, S. N. Ehrlich, F. Y. Hansen, E. Lu, and P. Miceli, Chem. Phys. Lett. 377, 99 (2003).

${ }^{15}$ S. Trogisch, M. J. Simpson, H. Taub, U. G. Volkmann, M. Pino, and F. Y. Hansen, J. Chem. Phys. 123, 154703 (2005).

${ }^{16}$ U. G. Volkmann, M. Pino, L. A. Altamirano, H. Taub, and F. Y. Hansen, J. Chem. Phys. 116, 2107 (2002).

${ }^{17}$ T. Pfohl, D. Beaglehole, and H. Riegler, Chem. Phys. Lett. 260, 82 (1996).

${ }^{18}$ E. Sloutskin, Z. Sapir, C. D. Bain, Q. Lei, K. M. Wilkinson, L. Tamam, M. Deutsch, and B. M. Ocko, Phys. Rev. Lett. 99, 136102 (2007).

${ }^{19}$ V. Chevallier, D. Petitjean, V. Ruffer-Meray, and M. Dirand, Polymer 40, 5953 (1999)

${ }^{20}$ I. M. Tidswell, B. M. Ocko, P. S. Pershan, S. R. Wasserman, G. M. Whitesides, and J. D. Axe, Phys. Rev. B 41, 1111 (1990).

${ }^{21}$ E. Ramirez, Thesis, Physics Faculty, PUC, 2004

${ }^{22}$ U. G. Volkmann, H. Mannebach, and K. Knorr, Langmuir 14, 4904 (1998).

${ }^{23}$ M. Bai, S. Trogisch, S. Magonov, and H. Taub, Ultramicroscopy 108, 946 (2008).

${ }^{24}$ R. Köhler, P. Lazar, and H. Riegler, Appl. Phys. Lett. 89, 241906 (2006).

${ }^{25}$ F. L. McCrackin, E. Passaglia, R. R. Stromberg, and H. L. Steinberg, J. Res. Natl. Bur. Stand., Sect. A, 67A, 363 (1963).

${ }^{26}$ R. M. A. Azzam and N. M. Bashara, Ellipsometry and Polarized Light (North-Holland, Elsevier Science, Amsterdam, 1992).

${ }^{27}$ H. G. Tompkins, A User's Guide to Ellispometry (Academic, San Diego, 1993).

${ }^{28}$ B. M. Law and D. Beaglehole, J. Phys. D 14, 115 (1981).

${ }^{29}$ J. W. O. Faul, U. G. Volkmann, and K. Knorr, Surf. Sci. 227, 390 (1990).

${ }^{30}$ N. W. Ashcroft and N. David Mermin, Solid State Physics (Saunders College, Philadelphia, 1976), p. 542.

${ }^{31}$ M. J. Dignam and J. Fedyk, J. Phys. (France) 38, C5 (1977).

${ }^{32}$ L. Knufing, H. Schollmeyer, H. Riegler, and K. Mecke, Langmuir 21, 992 (2005).

${ }^{33}$ F. Y. Hansen, K. W. Herwig, B. Matthies, and H. Taub, Phys. Rev. Lett. 83, 2362 (1999).

${ }^{34}$ F. Y. Hansen, L. Criswell, D. Fuhrmann, K. W. Herwig, A. Diama, R. M. Dimeo, D. A. Neumann, U. G. Volkmann, and H. Taub, Phys. Rev. Lett. 92, 046103 (2004)

${ }^{35}$ SPARTAN 08 , computational chemistry software for research and higher education, http://www.computational-chemistry.co.uk/.

${ }^{36}$ M. Maroncelli, H. L. Strauss, and R. G. Snyder, J. Chem. Phys. 82, 2811 (1985). 